\title{
Fakten gegen Behauptungspolitik: Und sie dreht sich doch
}

\section{Jürg Unger-Köppel}

Dr. med., Mitglied des FMH-Zentralvorstandes, Departementsverantwortlicher Stationäre Versorgung und Tarife

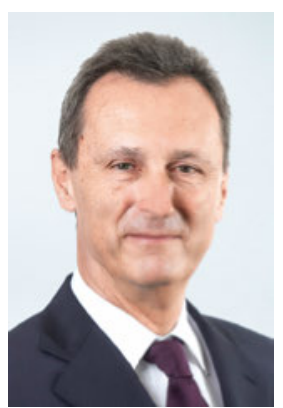

Es ist leicht, Behauptungen aufzustellen. Damit wird viel Politik gemacht. Diese Tendenz ist nicht neu. Lange wurde behauptet, dass die Erde eine Scheibe sei oder dass die Sonne um die Erde kreise. In neuerer Zeit wurde behauptet, dass die DRG zu bloody exits - blutigen Austritten - führen würden. Die angewandte Strategie ist einfach: Man möchte etwas erreichen und verkauft die gewünschte Massnahme als wirksame Medizin gegen das, was man behauptet. Je lauter man dabei schreit und je besser die Abbildung dieses Schreis in den Medien gelingt, desto schneller glauben alle, dass die Behauptung auch wahr sei. Über die Ärzteschaft und ihr Wirken wird immer wieder sehr viel behauptet, orchestriert mit lautem Aufschreien in den Medien. Dabei ist es meist einfach zu erkennen, welche Massnahmen mit den aufgestellten Behauptungen der Bevölkerung verkauft werden sollen. Gute Medizin beruht nicht auf Behauptungen, sondern auf sorgfältig erarbeiteten Tatsachen. Dies ist natürlich viel ressourcenintensiver, als einfach Behauptungen aufzustellen. Fakten gewinnt man mit wohl geplanten und aufwendig durchgeführten Untersuchungen. So gelingt der Fortschritt in der Medizin. Die gleiche Sorgfalt wendet die FMH an, um die Anliegen der Ärzteschaft in der Öffentlichkeit glaubwürdig vertreten zu

Die gleiche Sorgfalt wendet die FMH an, um die Anliegen der Ärzteschaft in der Öffentlichkeit glaubwürdig vertreten zu können.

können. Dazu braucht es sorgfältig erhobene Zahlen. Die FMH befragt zu diesem Zweck regelmässig die Ärzteschaft in den Spitälern der Somatik, der Psychiatrie und der Rehabilitation sowie aus der Praxis zu aktuellen Themen aus dem Gesundheitswesen, um die Erfahrungen von der Front des ärztlichen Handelns quantitativ zu erfassen.

Rohe Zahlen alleine können aber auch in die Irre führen. Wir kennen das Beispiel vom Rückgang der Stör- che in Norddeutschland und dem parallelen Geburtenrückgang. Deshalb müssen die Zahlen mittels geeigneter Fragen sorgfältig erhoben und die Antworten mit Bedacht ausgewertet werden. Das Departement Stationäre Versorgung und Tarife arbeitet aus diesem Grund mit dem Forschungsinstitut gfs.bern zusammen, um das medizinische Wissen und die Befragungsmethoden für die Auswertungen optimal zu koppeln.

Die jährliche Befragung stellt sicher, dass keine Zufallsresultate an die Öffentlichkeit gelangen, sondern gesichertes Wissen.

Unsere medizinischen Lehrer haben uns eingepaukt, dass ein überraschender Laborwert auch eine falsche Messung sein und damit einem Artefakt entsprechen kann. Also lernten wir, solche Werte zuerst zu überprüfen, bevor wir Massnahmen ergreifen durften. Dieselbe Methode wenden wir auch bei unserer Erhebung an. Durch die jährliche Wiederholung der Befragung wollen wir sicherstellen, dass wir keine Zufallsresultate generieren und an die Öffentlichkeit tragen, sondern gesichertes Wissen. So gewinnen wir nicht nur verlässliche Daten, sondern können auch Entwicklungen über die Zeit gut abbilden. Und ein Trend zeigt sich in unseren Resultaten, der besonders Besorgnis erregend ist: Die Ärztinnen und Ärzte beginnen langsam die Freude an ihrem Beruf zu verlieren. Umso wichtiger ist es deshalb, dass die erhobenen Zahlen ernst genommen werden und daraus die richtigen Schlüsse auch ausserhalb der Ärzteschaft gezogen werden, besonders in der Politik!

Weitere Resultate der neusten gfs.bern-Befragung der Ärzteschaft und den Vergleich mit den Vorjahren finden Sie in dieser Ausgabe der Schweizerischen Ärztezeitung auf Seite 45. 